\title{
Re: Significance of Atypical Urinary Cytology in the Evaluation of Patients with End-stage Renal Disease for Kidney Transplantation - A Retrospective Study
}

\author{
Law J1, Ali 01, Dobrin A2, Brar H1, Luke PP1,2, Sener A1,2,3 \\ 1 Western University Faculty of Medicine, Department of Urological Surgery, London, Canada \\ 2 Western University, Schulich Faculty of Medicine \& Dentistry, London, Canada \\ 3 Western University Faculty of Medicine, Department of Microbiology and Immunology, London, Canada
}

Transpl Int. 2019 May 17. doi: 10.1111/tri.13464

\section{EDITORIAL COMMENT}

Active malignancy is a contraindication to kidney transplantation (KT) since immunosuppression may exacerbate an underlying malignancy. In this single center retrospective cohort study, the authors aimed to evaluate the incidence of urothelial carcinoma (UC) in end-stage renal disease (ESRD) patients presenting for KT work up and establish the incidence of UC in ESRD patients with atypical urinary cytology. Out of 703 patients, 430 ESRD patients with sufficient urine had been screened with urinary cytology and those with atypical cytology (151patients, 35\%) or risk factors for bladder cancer had undergone cystoscopy. A total of 6 (0.85\%) patients (only 3 with atypical cytology) were found to have bladder cancer and treated with transurethral resection of the bladder and eventually underwent KT. One patient, who had been diagnosed with high-grade non-muscle invasive bladder cancer during double-J stent removal after KT, progressed to metastatic disease. The rate of atypical cytology in ESRD population in this study (35\%) was high compared to that in the general population, which has been reported as low as 1.9\%. However, lack of high-grade lesions in this study makes it hard to exclude urinary cytology in the evaluation of these patients. The results of this study indicate that the incidence of bladder cancer is higher in ESRD patients compared to that in the general population, however, in this population, atypical cytology alone should not be an indication for cystoscopy.

Yarkın Kamil Yakupoglu, MD

oCopyright 2019 by the Association of Urological Surgery / Journal of Urological Surgery published by Galenos Publishing House. 